\title{
Diabetic kidney disease: the onset of a new era?
}

\section{Paola Fioretto ${ }^{1}$}

Published online: 9 September 2020

(c) Italian Society of Nephrology 2020
Diabetic kidney disease (DKD) is the most common cause of end-stage renal disease (ESRD) worldwide [1, 2], affecting approximately $40 \%$ of patients with diabetes. DKD in both type 1 and type 2 diabetes is associated with increased mortality and cardiovascular risk; indeed the majority of patients with DKD die of cardiovascular complications before reaching ESRD [3].

Thus, DKD is a major burden for the person with diabetes and also for the health care systems. Currently, there is no reliable test to predict who will develop DKD and by the time increased albuminuria and/or reduction in GFR are detected the burden of renal and cardiovascular risk is already very high. Better control of glycemia and blood pressure, including renin-angiotensin system blockade (RASB) $[4,5]$, are effective in slowing DKD progression rate but have failed to substantially decrease the annual incidence of new cases of ESRD due to diabetes. Despite multifactorial interventions, including tight control of glucose, blood pressure, lipids and lifestyle modifications, the residual risk of progression towards ESRD remains high among patients with type 2 diabetes [6].

Thus, there is an unmet need for better treatment options, starting as early as possible to prevent the development and slow progression of DKD in high risk patients. This sad scenario is likely to rapidly change with the use of new glucose-lowering classes of drugs that have been shown to have nephroprotective effects. In particular SGLT2 inhibitors, as a class, reduce GFR loss and ESRD, as demonstrated in cardiovascular outcome trials, where this class of drugs significantly decreased major adverse cardiovascular events, hospitalization for heart failure, and renal events [7-9]. The nephroprotective effects have been confirmed in 'renal' studies performed in patients with advanced CKD (CREDENCE trial, performed in patients with type 2 diabetes [10], and in

Paola Fioretto

Paola.fioretto@unipd.it

1 Department of Medicine, Clinica Medica 3, University of Padova, Via Giustiniani 2, 35128 Padova, Italy
DAPA-CKD, performed in both non diabetic and diabetic patients [11], and stopped early in March 2020 after overwhelming efficacy. The results of this trial will be presented at the European Heart Association meeting on August 31st). SGLT2 inhibitors, in addition to slowing GFR loss, have a potent antiproteinuric effect, which is additive to that provided by RAS blockade. In patients with abnormal albuminuria at baseline, all trials have shown a significant reduction in urine albumin to creatinine ratio (UACr) levels of about 30-40\% [7-10]. Thus, SGLT2 inhibitors undoubtedly substantially slow progression towards ESRD in patients with DKD; the question is whether these agents may completely change the natural history of renal complications in diabetes and whether they are effective also in primary prevention. A reduction in the risk of developing new onset microalbuminuria was observed in CANVAS and DECLARE-TIMI [8, 12]. Recently the CVD-REAL3 study, performed in 70,000 patients with normal baseline GFR $\left(91 \mathrm{ml} / \mathrm{min} / 1.73 \mathrm{~m}^{2}\right)$ from five countries demonstrated that the use of SGLT2 inhibitors was associated with less GFR loss and renal endpoints [12], thus supporting the efficacy of this class in primary prevention. Therefore, it is likely that soon these drugs will be recommended in all patients with type 2 diabetes to prevent the onset and progression of DKD [13].

Despite the enthusiasm in the scientific community derived from the strong nephroprotective effects of SGLT2 inhibitors, it is important to keep in mind that the antialbuminuric response to SGLT2 inhibition markedly varies among patients, [14] and that only $65 \%$ of patients had a substantial reduction in albuminuria. This variability supports the concept that there are responders and non-responders to SGLT2 inhibitors.

Another class of glucose-lowering agents, GLP1 RA, have been shown to have nephroprotective effects, affecting predominantly albuminuria.

Now that the renal results of the cardiovascular outcome trials with SGLT2 inhibitors have been confirmed in two large renal trials, it is tempting to propose that we are at 'the onset of a new era' in the management of DKD (and likely also of CKD in non diabetic patients). 
Thus, the idea of the Editor of the Journal of Nephrology to have a Special issue dedicated to DKD was timely and highly appreciated.

In this Special Issue of the Journal we provide an up to date view of different aspects of DKD.

This issue starts with an overview of renal structure in type 2 diabetes, emphasizing the heterogeneity of the lesions underlying renal dysfunction and the relevance of performing kidney biopsies in these patients. We propose that this heterogeneity in renal lesions is likely to account for the different responses to treatments in individual patients.

The identification of patients at high risk of DKD is currently imprecise using the classic markers of renal function, and thus more precise biomarkers are needed. Novel omicsbased biomarkers for the initiation, presence and progression of DKD and the expanding knowledge of the biochemical pathways involved, possibly indicating potential targets for future therapies, are described in the second article. The following two articles describe potential mechanisms of renal damage, including the role of dicarbonyls, Advaced Glycation End-Products (AGEs) and oxidative stress in DKD.

Three articles consider important risk factors for DKD and the effects of their treatment: blood pressure, lipids and uric acid. Finally, the last reviews focus on the effects of GLP1 RA and SGLT2 inhibitors on the kidney.

I would like to express my gratitude to the authors contributing to this issue, and I hope that the reader will find this collection of articles of interest.

\section{Compliance with ethical standards}

Conflict of interest PF received personal fees from AstraZeneca, Boehringer Ingelheim, Mundipharma, Lilly, Novartis.

Ethical approval This article does not contain any studies with human participants or animals.

Informed consent For this type of study formal consent is not required.

\section{References}

1. https://www.usrds.org/2019/view/USRDS_2019_ES_final.pdf
2. https://www.era-edta-reg.org/files/annualreports/pdf/AnnRep2016 .pdf

3. Chronic Kidney Disease Prognosis Consortium, Matsushita K, van der Velde M et al (2010) Association of estimated glomerular filtration rate and albuminuria and all-cause and cardiovascular mortality in genetral population cohorts: a collaborative metaanalysis. Lancet 375:2073-20181

4. Brenner BM, Cooper ME, de Zeeuw D et al (2001) RENAAL Study Investigators. Effects of losartan on renal and cardiovascular outcomes in patients with type 2 diabetes and nephropathy. $\mathrm{N}$ Engl J Med 345:861-869

5. Lewis EJ, Hunsicker LG, Bain RP et al (1993) The effect of angiotensin-converting-enzyme inhibition on diabetic nephropathy. The Collaborative Study Group. N Engl J Med 329:1456-1462

6. Fioretto P, Dodson PM, Ziegler D, Rosenson RS (2010) Residual microvascular risk in diabetes: unmet needs and future directions. Nat Rev Endocrinol 6:19-25

7. Wanner C, Inzucchi SE, Lachin JM et al (2016) Empagliflozin and progression of kidney disease in Type 2 Diabetes. N Engl J Med 375:323-334

8. Neal B, Perkovic V, Mahaffey KW et al (2017) Canagliflozin and cardiovascular and renal events in type 2 diabetes. $\mathrm{N}$ Engl J Med 377:644-657

9. Mosenzon O, Wiviott SD, Cahn A et al (2019) Effects of dapagliflozin on development and progression of kidney disease in patients with type 2 diabetes: an analysis from the DECLARETIMI 58 randomised trial. Lancet Diabetes Endocrinol 7:606-617

10. Perkovic V, Jardine MJ, Neal B et al (2019) Canagliflozin and renal outcomes in type 2 diabetes and nephropathy. N Engl J Med 380:2295-2306

11. Heerspink HJL, Stefansson BV, Chertow GM et al (2020) Rationale and protocol of the dapagliflozin and prevention of adverse outcomes in chronic kidney disease (DAPA-CKD) randomized controlled trial. Nephrol Dial Transplant 35(2):274-282. https:// doi.org/10.1093/ndt/gfz290

12. Raz I, Wiviott SD, Yanuv I et al. (2019) Effects of dapagliflozin on the urinary albumin-to-creatinine ratio in patients with type 2 diabetes: a predefined analysis from the DECLARE-TIMI 58 randomised, placebo-controlled trial. Presented at: American Diabetes Association 79th Scientific Sessions, San Francisco, CA, 7-11 June 2019 (abst 244)

13. Heerspink HJL, Karasik A, Thuresson M et al (2020) Kidney outcomes associated with sodium-glucose cotransporter-2 inhibition in real-world clinical practice: CVD-REAL 3, a multinational observational cohort study. Lancet Diabetes Endocrinol 8:27-35

14. Fioretto P, Vettor R, Pontremoli R (2020) SGLT2 inhibitors to prevent diabetic kidney disease. Lancet Diabetes Endocrinol 8:4-5

Publisher's Note Springer Nature remains neutral with regard to jurisdictional claims in published maps and institutional affiliations. 